\title{
A REMARK ON THE GLOBAL EMBEDDING PROBLEM FOR THREE-DIMENSIONAL CR MANIFOLDS
}

\author{
DAVID E. BARRETT
}

(Communicated by Irwin Kra)

\begin{abstract}
Examples are constructed of compact strictly pseudoconvex real analytic three-dimensional CR manifolds which admit no nonconstant CR functions. Also, the embedding problem for certain CR manifolds is shown to be related to a result of Hadamard on immersed convex hypersurfaces in $\mathbf{R}^{n}$.
\end{abstract}

Let $M$ be a compact strictly pseudoconvex CR manifold. We wish to consider the problem of whether or not $M$ admits a CR embedding into complex Euclidean space $\mathbb{C}^{n}$ for some $n$; this amounts to asking whether or not $M$ has a sufficiently rich collection of global CR functions. Boutet de Monvel has shown that the answer to this question is always positive when $\operatorname{dim} M \geq 5$ [BM]. In the case $\operatorname{dim} M=3$ it is known that in general $M$ may not be even locally embeddable [N] (see also [JT, $\mathbf{K u}$ ); furthermore, examples have been given which show that even when the CR structure on $M$ is real analytic so that the local problem is solvable it can happen that the global CR functions on $M$ fail to separate points of $M$ [B] (see also [R, AS]).

The purpose of this note is to present a very simple class of examples of threedimensional strictly pseudoconvex compact real analytic CR manifolds which are not globally embeddable into any $\mathbb{C}^{n}$. Indeed, most of these examples do not admit any nonconstant global CR functions; it seems reasonable to guess that this situation is typical also in greater generality.

The examples are presented in $\S 1$ below. In $\S 2$ we see that our inability to produce higher-dimensional examples by the same technique is related to a result of Hadamard which states that any immersed compact hypersurface in $\mathbb{R}^{n}(n \geq 3)$ with positive principal curvatures must actually be embedded [S, III, pp. 94-96, IV, pp. 121-122].

Examples similar to those presented below were known earlier to Harvey and Lawson (for nonpseudoconvex CR manifolds) [HL] and to Bedford and Dadok (for complex manifolds) [BD]. Also, I wish to thank C. Epstein for helpful conversations.

1. CR structures on $\mathrm{T}^{3}$. Let $\left(x_{1}, x_{2}, x_{3}\right)$ be coordinates on the 3 -torus $\mathbb{T}^{3}=$ $(\mathbb{R} / 2 \pi \mathbb{Z})^{3}$. A CR structure on $\mathbb{T}^{3}$ which is invariant under rotations of the first two coordinates is given by a complex vector field

$$
\bar{L}=\alpha\left(x_{3}\right) \frac{\partial}{\partial x_{1}}+\beta\left(x_{3}\right) \frac{\partial}{\partial x^{2}}+\gamma\left(x_{3}\right) \frac{\partial}{\partial x_{3}}
$$

Received by the editors December 16, 1986.

1980 Mathematics Subject Classification (1985 Revision). Primary 32F30.

Key words and phrases. CR manifolds, CR functions. 
such that $\bar{L}$ and its conjugate $L$ are nowhere $\mathbb{C}$-linearly dependent. If $\gamma(p)=0$ for some point $p$ then $\left\{x \in \mathbb{T}^{3}: x_{3}=p\right.$ \} is a compact complex curve; thus if we wish to examine only strictly pseudoconvex structures on $\mathbb{T}^{3}$ we may assume that $\gamma$ is nowhere vanishing. Replacing $\bar{L}$ by $\gamma^{-1} \bar{L}$ we may, in fact, assume that $\gamma$ is identically equal to one; then $\bar{L}$ gives a CR structure if and only if $\operatorname{Im} \alpha$ and $\operatorname{Im} \beta$ never vanish jointly.

Such a CR structure on $\mathbb{T}^{3}$ induces in a natural way a CR structure on the covering space $\mathbb{T}^{2} \times \mathbb{R}$. This covering space admits a $C R$ immersion into $\mathbb{C}^{2}$ by means of the coordinate functions

$$
z_{1}=\exp \left\{i\left(x_{1}-\int_{0}^{x_{3}} \alpha(t) d t\right)\right\}, \quad z_{2}=\exp \left\{i\left(x_{2}-\int_{0}^{x_{3}} \beta(t) d t\right)\right\}
$$

indeed, $\bar{L} z_{1}=\bar{L} z_{2}=0$, and at each point one of the forms

$$
d z_{1} \wedge d \bar{z}_{1} \wedge d z_{2}=2\left|z_{1}\right|^{2} z_{2}(\operatorname{Im} \alpha) d x_{1} \wedge d x_{2} \wedge d x_{3}
$$

or

$$
d z_{1} \wedge d z_{2} \wedge d \bar{z}_{2}=-2 z_{1}\left|z_{2}\right|^{2}(\operatorname{Im} \beta) d x_{1} \wedge d x_{2} \wedge d x_{3}
$$

must be nonzero.

Note that we can recover the original CR structure on $T^{3}$ by taking the portion of the immersed hypersurface which corresponds to $T^{2} \times(0,2 \pi+\varepsilon)$ and gluing the portion corresponding to $\pi^{2} \times(0, \varepsilon)$ to the portion corresponding to $\mathbb{T}^{2} \times(2 \pi, 2 \pi+\varepsilon)$. The formulas for $z_{1}$ and $z_{2}$ show that the gluing map is of the form

$$
\left(z_{1}, z_{2}\right) \mapsto\left(A z_{1}, B z_{2}\right)
$$

where $A=\exp \left\{-i \int_{0}^{2 \pi} \alpha(t) d t\right\}$ and $B=\exp \left\{-i \int_{0}^{2 \pi} \beta(t) d t\right\}$.

Representing our immersed hypersurface in the logarithmic coordinates familiar from the study of Reinhardt domains we may visualize the situation as shown in Figure 1. The dotted lines represent translation by the vector $(\log |A|, \log |B|)$; the diagram suppresses the rotation factors $\arg A$ and $\arg B$.

Conversely, given any immersed curve in $\mathbb{R}^{2}$ which can be pasted together by a translation as in Figure 1 we can paste together the corresponding $T^{2}$-invariant immersed hypersurface in $\mathbb{C}^{2}$ to obtain a compact CR manifold; $\log |A|$ and $\log |B|$ are determined by our choice of curve but $\arg A$ and $\arg B$ may be chosen arbitrarily.

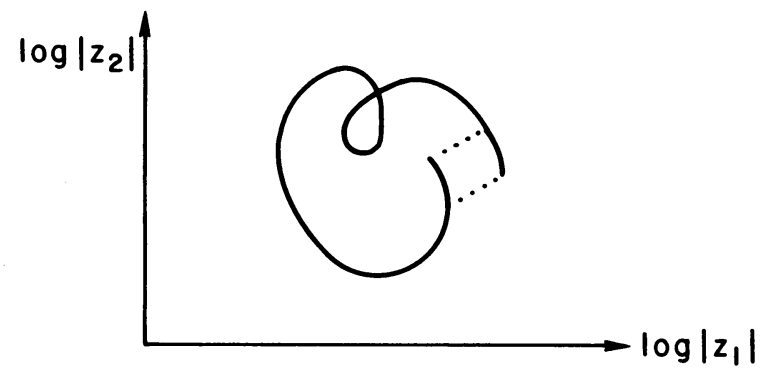


We note that the CR structure given by $\bar{L}$ is strictly pseudoconvex if and only if

$$
\left.\langle[L, \bar{L}] \wedge L] \wedge \bar{L}, d x_{1} \wedge d x_{2} \wedge d x_{3}\right\rangle=-4\left(\operatorname{Im} \alpha \operatorname{Im} \beta^{\prime}-\operatorname{Im} \beta \operatorname{Im} \alpha^{\prime}\right)
$$

is nowhere vanishing. Geometrically this is, of course, equivalent to the positivity of the curvature of the curve in Figure 1, as we may easily check by noting that the tangent slopes of this curve are given by $\operatorname{Im} \beta / \operatorname{Im} \alpha$.

From either point of view it is easy to see that there are many real analytic strictly pseudoconvex CR structures realizing any given nonzero choices of $A$ and $B$.

Clearly if $A=B=1$ then we have constructed a CR immersion of $\mathbb{T}^{3}$ into $\mathbb{C}^{2}$. What happens if $A \neq 1$ or $B \neq 1$ ? We note that any CR function $u$ on $\mathbb{T}^{3}$ admits a Fourier expansion

$$
u=\sum_{m, n \in \mathbb{Z}} u_{m n}
$$

where $u_{m n}$ is of the form $e^{i\left(m x_{1}+n x_{2}\right)} \phi_{m n}\left(x_{3}\right)$. The usual "variation of parameters" argument shows that $\phi_{m n}$ must solve the differential equation

$$
i(m \alpha+n \beta) \phi_{m n}+\phi_{m n}^{\prime}=0
$$

it follows that each $u_{m n}$ must be of the form $c_{m n} z_{1}^{m} z_{2}^{n}$ for some constants $c_{m n}$. Since $u_{m n}$ is well defined on $\mathbb{T}^{3}$ we must have $c_{m n}=0$ whenever $A^{m} B^{n} \neq 1$.

Let $\Lambda$ denote the lattice $\left\{(m, n) \in \mathbb{Z}^{2}: A^{m} B^{n}=1\right\}$, and let $\Lambda_{\mathbf{R}}$ denote the subspace of $\mathbb{R}^{2}$ spanned by $\Lambda$. Generically we have $\operatorname{dim} \Lambda_{\mathbf{R}}=0$ are all global CR functions are constant. If $\operatorname{dim} \Lambda_{\mathbb{R}}=1$, then there exist nonconstant global $\mathrm{CR}$ functions but they are all functionally dependent on a single generating function. Finally, if $\operatorname{dim} \Lambda_{\mathbb{R}}=2$ then any pair of CR functions corresponding to linearly independent elements of $\Lambda$ give rise to CR immersion of $\mathbb{T}^{3}$ into $\mathbb{C}^{2}$. If $\Lambda \neq \mathbb{Z}^{2}$, however, global CR functions will fail to separate points of any two-torus $\{x \in$ $\mathrm{T}^{3} ; x_{3}=$ constant $\}$.

Let us assume now that $\Lambda=\mathbb{Z}^{2}$, i.e., that $A=B=1$. Then the curve in Figure 1 is actually a closed curve representing an immersed hypersurface in $\mathbb{C}^{2}$. If the curve has a self-intersection then consideration of the Fourier expansion used above shows that CR functions again fail to separate points of $\pi^{3}$. Thus our immersed hypersurface is embeddable if and only if the curve in Figure 1 is a simple closed curve; in the pseudoconvex case this amounts to assuming that the rotation index

$$
R=(2 \pi)^{-1} \int_{0}^{2 \pi} \frac{\operatorname{Im} \alpha \operatorname{Im} \beta^{\prime}-\operatorname{Im} \beta \operatorname{Im} \alpha^{\prime}}{(\operatorname{Im} \alpha)^{2}+(\operatorname{Im} \beta)^{2}} d x_{3}
$$

is equal to \pm 1 (see [S, II. 1.24]).

The space of all strictly pseudoconvex $\mathbb{T}^{2}$-invariant CR structrures on $\mathbb{T}^{3}$ has countably many components corresponding to the distinct nonzero integer values of $R$. The embeddable structures form a submanifold of real codimension four (given by the complex equations $A=B=1$ ) contained inside the components with $R= \pm 1$. We note that these structures are precisely the ones for which the differential operator $\bar{L}$ has closed range as a partially defined operator from $L^{2}\left(T^{3}\right)$ to $L^{2}\left(\mathbb{T}^{3}\right)[\mathbf{K o}]$.

Taking a slightly different point of view we observe that the arguments above show that a CR structure of the sort we have considered is embeddable if and 
only if it is embeddable equivariantly with respect to the standard action of $\mathbb{T}^{2}$ on $\mathbb{C}^{2}$. Such an embedding is always possible locally in the $x_{3}$-variable and is well determined up to the choice of two dilation factors in $\mathbb{C}^{*}$. Choosing a family of local embeddings, the discrepancies along overlaps give rise to a Čech one-cycle with values in $\mathbb{C}^{*} \times \mathbb{C}^{*}$ (see Figure 2 ). The $\mathrm{CR}$ structure is equivariantly immersable if and only if the corresponding cohomology class in $H^{1}\left(S^{1}, \mathbb{C}^{*} \times \mathbb{C}^{*}\right)$ is trivial.

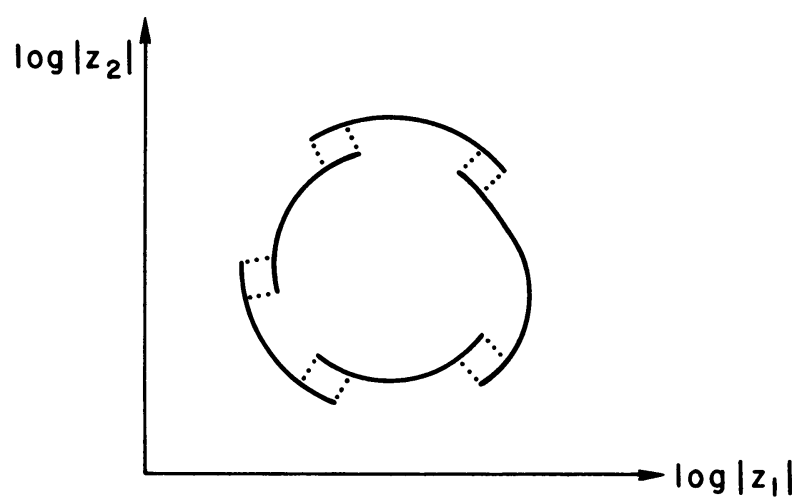

FIGURE 2

2. Higher dimensions. In analogy to our work above we may consider $\mathbb{T}^{n}$ invariant CR structures on $\mathbb{T}^{n} \times S$, where $S$ is a compact manifold of real dimension $n-1$. If $n \geq 3$ and the structure is strictly pseudoconvex, then we know from the theorem of Boutet de Monvel cited earlier that the structure is embeddable into some $\mathbb{C}^{n}$. In this special case we can give an elementary proof which is outlined below.

THEOREM. Let $S$ be a compact manifold of real dimension $n-1$ with $n \geq 3$ and let $M=\mathrm{T}^{n} \times S$ be equipped with a $\mathbb{T}^{n}$-invariant strictly pseudoconvex $C R$ structure. Then $S$ is diffeomorphic to the sphere $S^{n-1}$ and $M$ is $C R$ isomorphic to the boundary of a strictly pseudoconvex Reinhardt domain in $\left(\mathbb{C}^{*}\right)^{n}$.

Outline of proof. The $\mathrm{T}^{n}$-invariance of the CR structure means that the CR structure is generated by vector fields of the form

$$
\bar{L}=\sum_{j=1}^{n} a_{j}(\rho) \frac{\partial}{\partial x_{j}}+X,
$$

where $\rho$ denotes points in $S$, the $x_{j}$ are standard coordinates on $\mathrm{T}^{n}$, and $X$ is a vector field on $S$. Thus a function of the form

$$
f_{j}(x, p)=e^{i x_{j}} u_{j}(\rho)
$$

is CR if and only if it satisfies a family of equations of the form

$$
i a_{j}(\rho) u_{j}(\rho)+X u_{j}(\rho)=0 .
$$


It is not hard to check that the strict pseudoconvexity hypothesis guarantees that we have exactly one such equation for each $j$ and for each vector field $X$ on $S$ so that each $u_{j}$ must satisfy a total differential equation $d \log u_{j}=\alpha_{j}$; the integrability conditions on the CR structure guarantee that each $\alpha_{j}$ is a closed 1 -form.

Thus we may solve locally for the $u_{j}$ to obtain an equivariant immersion of $\mathbb{T}^{n} \times U$ into $\mathbb{C}^{n}$ for any simply-connected open subset $U$ of $S$. The corresponding immersion

$$
\Lambda_{U}: \rho \mapsto\left(\log \left|u_{1}(\rho)\right|, \ldots, \log \left|u_{n}(\rho)\right|\right)
$$

of $U$ into logarithmic coordinate space $\mathbb{P}^{n}$ is determined up to additive constants so that we have induced a well-defined Gauss map from $S$ to the unit sphere $S^{n-1}$. The strict pseudoconvexity hypothesis implies that the image of each $\Lambda_{U}$ has positive principal curvatures so that the Gauss map is a local diffeomorphism from $S$ to $S^{n-1}$; since $S$ is a compact and $S^{n-1}$ is simply-connected the Gauss map is in fact a diffeomorphism of $S$ onto $S^{n-1}$. In particular, $S$ is simply connected, so that we have a well-defined map

$$
\Lambda: \rho \mapsto\left(\log \left|u_{1}(\rho)\right|, \ldots, \log \left|u_{n}(\rho)\right|\right)
$$

from $S$ to $\mathbb{R}^{n}$. The image of $\Lambda$ is a compact immersed hypersurface with positive principal curvatures; thus the result of Hadamard cited in the introduction implies that $\Lambda$ is an embedding. Thus $\Lambda(S)$ bounds a strictly convex open subset of logarithmic coordinate space $\mathbb{R}^{n}$ and the boundary of the corresponding Reinhardt domain is CR isomorphic to $M$.

\section{REFERENCES}

[AS] A. Andreotti and Y.-T. Siu, Projective embedding of pseudoconcave spaces, Ann. Scuola Norm. Sup. Pisa 24 (1970), 231-278.

[B] D. Burns, Global behavior of some tangential Cauchy-Riemann equations, Partial Differential Equations and Geometry (Proc. Conf., Park City, Utah), Dekker, 1979.

[BD] E. Bedford and J. Dadok, personal communication.

[BM] L. Boutet de Monvel, Intégration des equation de Cauchy-Riemann induites formelles, Séminaire Goulaoic-Lions-Schwartz, Exposé IX, 1974-1975.

[HL] R. Harvey and B. Lawson, On boundaries of complex analytic varieties. I, Ann. of Math. (2) 102 (1975), 233-290.

[JT] H. Jacobowitz and F. Treves, Non-realizable CR structures, Invent. Math. 66 (1982), 231-249.

[Ko] J. J. Kohn, Estimates for $\bar{\partial}_{b}$ on pseudo-convex CR manifolds, Proc. Sympos. Pure Math., vol. 43, Amer. Math. Soc., Providence, R.I., 1985.

[Ku] M. Kuranishi, Strongly pseudoconvex CR structures over small balls, I, II, Ann. of Math. (2) 115 (1982), 451-500; 116 (1982), 1-64.

[N] L. Nirenberg, On a problem of Hans Lewy, Uspekhi Mat. Nauk 29 (1974), 241-251.

[R] H. Rossi, Attaching analytic spaces to an analytic space along a pseudoconcave boundary, Proc. Conf. Complex Analysis (Minneapolis), Springer-Verlag, Berlin, Heidelberg, and New York, 1965.

[S] M.Spivak, A comprehensive introduction to differential geometry (5 volumes), Publish or Perish, 1975 . 08544

Department of Mathematics, Princeton University, Princeton, New Jersey

Current address: Department of Mathematics, University of Michigan, Ann Arbor, Michigan 48109 